\title{
GENERALITY OF HENSTOCK-KURZWEIL TYPE INTEGRAL ON A COMPACT ZERO-DIMENSIONAL METRIC SPACE
}

\author{
Francesco Tulone
}

\begin{abstract}
A Henstock-Kurzweil type integral on a compact zero-dimensional metric space is investigated. It is compared with two Perron type integrals. It is also proved that it covers the Lebesgue integral.
\end{abstract}

In [8] a derivation basis and a Henstock-Kurzweil type integral with respect to this basis were introduced on a compact zero-dimensional metric space $X$ and this integral was applied to some problems in harmonic analysis. Moreover, it was stated that the results obtained there are also true for the case of the Lebesgue integral. To justify this announcement, we have to check that Lebesgue integral is covered by the above mentioned Henstock-Kurzweil type integral.

One of the aims of this paper is to give this justification. To this end we investigate generality of this integral. We prove that Lebesgue integral is included in our Henstock-Kurzweil type integral using Perron approach. We define two Perron type integrals and we get that under certain assumption they are equivalent to the Henstock-Kurzweil type integral.

These results are known for the corresponding integrals with respect to the usual interval basis on the real line (see [3] and [4]).

We remind some notions and notations from 8 . We suppose that a sequence $\left\{C_{n}\right\}_{n=1}^{\infty}$ of covers of a compact zero-dimensional metric space $X$ is given such that

(a) for each fixed $n$, elements $K_{j}^{(n)}$ of $C_{n}$ are disjoint and clopen;

(b) each element of $C_{n}$ is properly contained in some element of $C_{n-1}$, for $n \geq 2$

(c) $C_{1}=\{X\}$;

(d) $\bigcup_{n=1}^{\infty} C_{n}$ is a base for the topology of $X$.

(C) 2011 Mathematical Institute, Slovak Academy of Sciences. 2010 Mathematics Subject Classification: 26A39.

Keyw ords: Henstock-Kurzweil integral, Perron integral, Lebesgue integral, derivation basis, compact zero-dimensional metric space, major and minor function. 


\section{FRANCESCO TULONE}

As $X$ is compact, the cover $C_{n}$ is finite for each $n \in \mathbb{N}$. Let $C_{n}=\left\{K_{j}^{(n)}\right\}_{j=1}^{m(n)}$.

For each $x \in X$ and $n \in \mathbb{N}$ we denote by $K(n, x)$ the (unique) element $K_{j(n, x)}^{(n)}$ of $C_{n}$ containing $x$. Then a unique sequence $\{K(n, x)\}_{n}$ is defined for each $x$ so that (in view of $(\mathrm{d})$ )

$$
\bigcap_{n} K(n, x)=\{x\}
$$

We also assume that a Borel probability measure $\mu$ is given on $X$. Then for each fixed $n$ we have

$$
\sum_{j=1}^{m(n)} \mu\left(K_{j}^{(n)}\right)=1 .
$$

This measure can be extended in a usual way to be a complete measure on $X$. It is known that this type of measure being a completion of a Borel measure is regular (see [1]).

Now, having fixed the sequence $\left\{C_{n}\right\}_{n=1}^{\infty}$ and the measure $\mu$, we define a derivation basis $\mathcal{B}$ in $X$. Take any function $\nu: X \rightarrow \mathbb{N}$ and define a basis set by

$$
\beta_{\nu}:=\{(I, x): x \in X, I=K(n, x), n \geq \nu(x)\} .
$$

So our basis $\mathcal{B}$ in $X$ is a family of basis sets $\left\{\beta_{\nu}\right\}_{\nu}$ where $\nu$ runs over the set of all natural-valued functions on $X$. We denote by $\mathcal{I}$ the union $\bigcup_{n=1}^{\infty} C_{n}$ and we refer to elements of $\mathcal{I}$ as $\mathcal{B}$-intervals.

This basis has all the usual properties of a general derivation basis (see [5, [9]). First of all, it has the filter base property:

1) $\emptyset \notin \mathcal{B}$;

2) for every $\beta_{\nu_{1}}, \beta_{\nu_{2}} \in \mathcal{B}$ there exists $\beta_{\nu} \in \mathcal{B}$ such that $\beta_{\nu} \subset \beta_{\nu_{1}} \cap \beta_{\nu_{2}}$ (it is sufficient to take $\left.\nu=\max \left\{\nu_{1}, \nu_{2}\right\}\right)$.

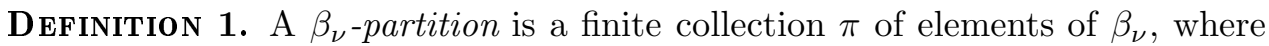
distinct elements $\left(I^{\prime}, x^{\prime}\right)$ and $\left(I^{\prime \prime}, x^{\prime \prime}\right)$ in $\pi$ have $I^{\prime}$ and $I^{\prime \prime}$ disjoint. If $L \in \mathcal{I}$ and

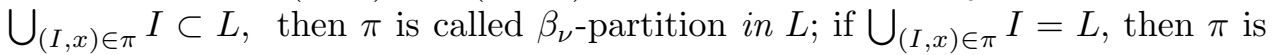
called $\beta_{\nu}$-partition of $L$.

Definition 2. We say that a basis $\mathcal{B}$ has the partitioning property if the following conditions hold:

i) for each finite collection $I_{0}, I_{1}, \ldots, I_{n}$ of $\mathcal{B}$-intervals with $I_{1}, \ldots, I_{n} \subset I_{0}$ and $I_{i}, i=1,2, \ldots, n$, being pairwise disjoint, the difference $I_{0} \backslash \bigcup_{i=1}^{n} I_{i}$ can be expressed as a finite union of pairwise disjoint $\mathcal{B}$-intervals;

ii) for each $\mathcal{B}$-interval $L$ and for any $\beta_{\nu} \in \mathcal{B}$ there exists a $\beta_{\nu}$-partition of $L$.

As we noticed in [8, the partitioning property of our basis $\mathcal{B}$ is guaranteed by the compactness of each $\mathcal{B}$-interval and by the fact that any two $\mathcal{B}$-intervals $I^{\prime}$ and $I^{\prime \prime}$ are either disjoint or one of them is contained in the other one. 


\section{GENERALITY OF HENSTOCK-KURZWEIL TYPE INTEGRAL}

Note that in the case of the basis $\mathcal{B}$, given a point $x \in X$, any $\beta_{\nu}$-partition contains only one pair $(I, x)$ with this point $x$. For a set $E \subset X$ and $\beta_{\nu} \in \mathcal{B}$ we write

$$
\beta_{\nu}(E):=\left\{(I, x) \in \beta_{\nu}: I \subset E\right\} \quad \text { and } \quad \beta_{\nu}[E]:=\left\{(I, x) \in \beta_{\nu}: x \in E\right\} .
$$

Definition of a Henstock-Kurzweil type integral can be given for the basis $\mathcal{B}$ considered here, in the following form:

Definition 3. Let $L \in \mathcal{I}$. A real-valued function $f$ on $L$ is said to be Henstock-Kurzweil integrable with respect to the basis $\mathcal{B}$ (or $H_{\mathcal{B}}$-integrable) on $L$, with $H_{\mathcal{B}}$-integral $A$, if for every $\varepsilon>0$, there exists a function $\nu: L \rightarrow \mathbb{N}$ such that for any $\beta_{\nu}$-partition $\pi$ of $L$ we have:

$$
\left|\sum_{(I, x) \in \pi} f(x) \mu(I)-A\right|<\varepsilon .
$$

We denote the integral value $A$ by $\left(H_{\mathcal{B}}\right) \int_{L} f$.

It is easy to check, using regularity of the measure $\mu$, that a function which is equal to zero almost everywhere on $L \in \mathcal{I}$, is $H_{\mathcal{B}}$-integrable on $L$ with integral value equal to zero. So we can state

Lemma 1. If $f$ is $H_{\mathcal{B}}$-integrable on $X$ and $f=h$ almost everywhere, then $h$ is also $H_{\mathcal{B}}$-integrable and their integrals coincide.

This justifies the following extension of Definition 3 to the case of functions defined only almost everywhere on $L$.

Definition 4. A real-valued function $f$ defined almost everywhere on $L \in \mathcal{I}$ is said to be $H_{\mathcal{B}}$-integrable on $L$, with integral value $A$, if the function

$$
f_{1}(g):= \begin{cases}f(g), & \text { where } f \text { is defined, } \\ 0 & \text { otherwise, }\end{cases}
$$

is $H_{\mathcal{B}}$-integrable on $L$ to $A$ in the sense of Definition 3 ,

We note that if $f$ is $H_{\mathcal{B}}$-integrable on $L \in \mathcal{I}$, then it is $H_{\mathcal{B}}$-integrable also on any $\mathcal{B}$-interval $K \subset L$. So we can define the indefinite integral $F(K)=$ $\left(H_{\mathcal{B}}\right) \int_{K} f d \mu$. It can be checked that the indefinite $H_{\mathcal{B}}$-integral $F$ is an additive $\mathcal{B}$-interval function on the set of all $\mathcal{B}$-intervals $K \subset L$.

An essential part of the theory of Henstock-Kurzweil integral is based on the following proposition known as Kolmogorov-Henstock lemma.

LEMMA 2. If a function $f$ is $H_{\mathcal{B}}$-integrable on $L$, with $F$ being its indefinite $H_{\mathcal{B}}$ -integral, then for every $\varepsilon>0$, there exists $\beta_{\nu} \in \mathcal{B}$ such that for any $\beta_{\nu}$-partition $\pi$ in $L$ we have:

$$
\sum_{(I, x) \in \pi}|f(x) \mu(I)-F(I)|<\varepsilon
$$




\section{FRANCESCO TULONE}

P r o of. A proof can be obtained by adjusting the proof of a similar real line version lemma (see [3, Theorem 3.2.1] and [4, Lemma 3.9]).

Definition 5. Given a real-valued set function $F$ on $\mathcal{I}$, we define the upper and lower $\mathcal{B}$-derivative at a point $x$, with respect to the basis $\mathcal{B}$ and measure $\mu$, as

$$
\bar{D}_{\mathcal{B}} F(x):=\limsup _{n \rightarrow \infty} \frac{F(K(n, x))}{\mu(K(n, x))} \quad \text { and } \quad \underline{D}_{\mathcal{B}} F(x):=\liminf _{n \rightarrow \infty} \frac{F(K(n, x))}{\mu(K(n, x))},
$$

respectively. If $\bar{D}_{\mathcal{B}} F(x)=\underline{D}_{\mathcal{B}} F(x)$, then $F$ is set to be $\mathcal{B}$-differentiable at the point $x$ with $\mathcal{B}$-derivative, $D_{\mathcal{B}} F(x)$ denotes this common value.

The following theorem was proved in [8, Theorem 1].

TheOREM 1. If a function $f$ is $H_{\mathcal{B}}$-integrable on a $\mathcal{B}$-interval $L$, then the indefinite $H_{\mathcal{B}}$-integral $F(I)=\left(H_{\mathcal{B}}\right) \int_{I} f$, as an additive function on the set of all $\mathcal{B}$-subintervals of $L$, is $\mathcal{B}$-differentiable almost everywhere on $L$ and

$$
D_{\mathcal{B}} F(x)=f(x) \quad \text { a.e. on } L \text {. }
$$

The above theorem holds for every measure $\mu$. Some other properties, in particular continuity of the indefinite integral, depend on the fact whether $\mu$ is non-atomic or not.

Definition 6. A real-valued set function $F$ defined on $\mathcal{I}$ is $\mathcal{B}$-continuous at a point $x$ if

$$
\lim _{n \rightarrow \infty} F(K(n, x))=0 .
$$

Note that any non-atomic measure $\mu$ on $X$ is $\mathcal{B}$-continuous at any point.

It can be easily shown, using Lemma 2 , that the indefinite $H_{\mathcal{B}}$-integral on $L \in \mathcal{I}$ is $\mathcal{B}$-continuous at each point of $L$ if the measure $\mu$ is non-atomic. Indeed, fixing a point $x \in L$ we can use Lemma 2 for the partition in $L$ constituted by only one pair $(I, x)$ getting $|f(x) \mu(I)-F(I)|<\varepsilon$. We note that a non-atomic measure gives measure zero for any singleton, so using Lemma 1 we can put $f(x)=0$ without changing the value of integral, obtaining $|F(I)|<\varepsilon$ for any $(x, I) \in \beta_{\nu}$, i.e., the $\mathcal{B}$-continuity of $F$ at $x$.

Now we define a Perron type integral with respect to the basis $\mathcal{B}$.

Definition 7. Let $f$ be a point function on $X$. A $\mathcal{B}$-interval function $M$ (resp. $m$ ) is called a $\mathcal{B}$-major (resp. $\mathcal{B}$-minor) function of $f$ on $X$ if it is superadditive (resp. subadditive) and the lower (resp. upper) $\mathcal{B}$-derivative satisfies the inequality

$$
\underline{D}_{\mathcal{B}} M(x) \geq f(x) \quad\left(\text { resp. } \bar{D}_{\mathcal{B}} m(x) \leq f(x)\right) \quad \text { for all } \quad x \in X .
$$

A function $f$ is said to be $P_{\mathcal{B}}$-integrable if it has at least one $\mathcal{B}$-major and one $\mathcal{B}$-minor function and

$$
-\infty<\inf _{M}\{M(X)\}=\sup _{m}\{m(X)\}<+\infty,
$$




\section{GENERALITY OF HENSTOCK-KURZWEIL TYPE INTEGRAL}

where "inf" is taken over all $\mathcal{B}$-major functions $M$ and "sup" is taken over all $\mathcal{B}$-minor functions $m$. The common value is denoted by $\left(P_{\mathcal{B}}\right) \int_{X} f$ and is called $P_{\mathcal{B}}$-integral of $f$ on $X$.

It can be easily checked that for any $\mathcal{B}$-major function $M$ and for any $\mathcal{B}$-minor function $m$ we have $M(X) \geq m(X)$. This implies the correctness of the above definition.

In the same way we can define $P_{\mathcal{B}}$-integral on any $\mathcal{B}$-interval.

If in the above definition we assume all the $\mathcal{B}$-major and $\mathcal{B}$-minor functions to be $\mathcal{B}$-continuous, then we obtain the definition of $P_{\mathcal{B}}^{0}$-integral. It is clear that $P_{\mathcal{B}^{-}}^{0}$-integral is included in $P_{\mathcal{B}}$-integral.

Let $f$ be a $P_{\mathcal{B}^{-}}$-integrable function on $X$. Since $f$ is also integrable on each $\mathcal{B}$-interval $I \subset X$, we can define the indefinite integrals $F(I)=\left(P_{\mathcal{B}}\right) \int_{I} f$ and $F(I)=\left(P_{\mathcal{B}}^{0}\right) \int_{I} f$. It is easy to check that the indefinite integral $F$ is an additive $\mathcal{B}$-interval function on $\mathcal{I}$ in both cases.

In the standard way (see [5]) we can check that $H_{\mathcal{B}}$-integral is equivalent to the $P_{\mathcal{B}}$-integral. It follows in particular that Lemma 1 is also true for $P_{\mathcal{B}}$-integral. Because of this, an analogue of Definition 4 can be given for Perron integral in the case of functions defined only almost everywhere.

To compare $H_{\mathcal{B}}$-integral with $P_{\mathcal{B}}^{0}$-integral, we shall use the notion of variation.

Let $F$ be an additive set function on $\mathcal{I}, E$ an arbitrary fixed subset of $X$, and $A$ a $\mathcal{B}$-interval. For a fixed $\beta_{\nu} \in \mathcal{B}$, we set

$$
V_{\nu}(A)=V\left(E, F, \beta_{\nu}, A\right):=\sup \left\{\sum_{(I, g) \in \pi}|F(I)|: \pi \subset \beta_{\nu}[E] \cap \beta_{\nu}(A)\right\}
$$

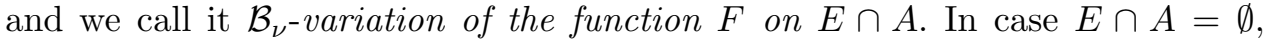
we define $V_{\nu}(A)=0$. For a fixed $E, V_{\nu}(A)$ is a non negative and superadditive interval function.

The next theorem can be proved for our basis $\mathcal{B}$ in a similar way as an analogous result in [7] for the particular case of zero-dimensional group.

TheORem 2. Let $F$ be a $\mathcal{B}$-continuous additive function defined on $\mathcal{I}$ with a finite value $V_{\nu}(X)$. Then for a fixed $E \subset X$ and a fixed function $\nu: X \rightarrow \mathbb{N}$ the $\mathcal{B}$-interval function $V_{\nu}(A)=V\left(E, F, \beta_{\nu}, A\right)$ is $\mathcal{B}$-continuous at each point $x \in X$.

As in [7, the above theorem can be used to construct $\mathcal{B}$-continuous major and minor functions for an $H_{\mathcal{B}}$-integrable function.

Theorem 3. Suppose that the measure $\mu$ on $X$ is non-atomic and a real-valued function $f$ is $H_{\mathcal{B}}$-integrable on $X$, with $F$ being its indefinite $H_{\mathcal{B}}$-integral. Then for any $\varepsilon>0$ there exist a $\mathcal{B}$-continuous $\mathcal{B}$-major function $M$ and a $\mathcal{B}$-continuous $\mathcal{B}$-minor function $m$ of $f$ such that

$$
M(X)-F(X)<\varepsilon \text { and } F(X)-m(X)<\varepsilon .
$$


Pr o of. As measure $\mu$ is non-atomic, the function $F$ is additive $\mathcal{B}$-continuous on $\mathcal{I}$.

Let $E=X \backslash C$ where $C=\left\{x \in X: D_{\mathcal{B}} F(x)=f(x)\right\}$. By Theorem 1, $\mu(E)=0$. Hence, as we have already mentioned, we can suppose $f(x)=0$ for all $x \in E$.

Having fixed $\varepsilon>0$, we can apply Lemma 2 to find a natural-valued function $\nu$ such that for any $\beta_{\nu}[E]$-partition $\pi$ we have

$$
\sum_{(I, x) \in \pi}|f(x) \mu(I)-F(I)|<\varepsilon .
$$

Since $f(x)=0$ if $x \in E$, this inequality and the notation (11) imply that

$$
V\left(E, F, \beta_{\nu}, X\right)<\varepsilon \text {. }
$$

By Theorem 2, the $\mathcal{B}$-interval function $V_{\nu}(A)=V\left(E, F, \beta_{\nu}, A\right)$ is $\mathcal{B}$-continuous at each point $x$ of $X$. Let

$$
M(A)=F(A)+V_{\nu}(A) \quad \text { and } \quad m(A)=F(A)-V_{\nu}(A)
$$

for each $\mathcal{B}$-interval $A \subset X$. Then the inequalities (2) follow from (3).

The rest of the proof follows the lines of the proof of [7, Theorem 3].

Using of the previous theorem we obtain for the case of non-atomic measure $\mu$ the following scheme

From this we obtain

$$
H_{\mathcal{B}} \subset P_{\mathcal{B}}^{0} \subset P_{\mathcal{B}}=H_{\mathcal{B}}
$$

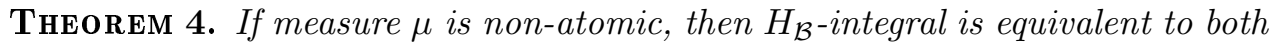
$P_{\mathcal{B}}$ and $P_{\mathcal{B}^{-}}^{0}$ integral.

In particular, we have got that for non-atomic measure $\mu$ and for our basis $\mathcal{B}$, the $P_{\mathcal{B}^{-}}^{0}$ and $P_{\mathcal{B}}$-integral are equivalent. We note that for a general basis the problem about the equivalence of the above Perron type integrals is still open (see [2]).

Now we turn to prove that our Henstock-Kurzweil type integral covers the Lebesgue integral.

We remind that a real valued function $f$ defined on a metric space is lower semi-continuous (upper semi-continuous) at $x_{0}$ if for every $\varepsilon>0$ there exists a neighborhood $U\left(x_{0}\right)$ of $x_{0}$ such that $f(x) \geq f\left(x_{0}\right)-\varepsilon$ (respectively $\left.f(x) \leq f\left(x_{0}\right)+\varepsilon\right)$ for all $x \in U\left(x_{0}\right)$. Equivalently, this can be expressed as $\liminf \operatorname{in}_{x \rightarrow x_{0}} f(x) \geq f\left(x_{0}\right)$ (respectively $\lim \sup _{x \rightarrow x_{0}} f(x) \leq f\left(x_{0}\right)$ ).

We need the following version of Vitali-Carathéodory theorem which is proved in [6. Chapter III, Theorem 7.6] for functions defined on $\mathbb{R}^{m}$, however, the same proof can be used for functions defined on any compact metric space with a regular measure on it. 


\section{GENERALITY OF HENSTOCK-KURZWEIL TYPE INTEGRAL}

TheOREM 5. Given a real-valued summable function $f$ on a compact metric space $X$ with a regular measure $\mu$ and any $\varepsilon>0$, there exist a summable lower semi-continuous function $l$ and a summable upper semi-continuous function $u$ such that

$$
\begin{gathered}
l(x) \geq f(x) \geq u(x) \quad \text { at each point } x \in X, \\
\int_{X}[l(x)-f(x)] d \mu<\varepsilon \quad \text { and } \quad \int_{X}[f(x)-u(x)] d \mu<\varepsilon .
\end{gathered}
$$

We finish with the following result.

TheоRем 6. Each real-valued summable function $f$ on a zero-dimensional compact metric space $X$ with a regular measure $\mu$ is $P_{\mathcal{B}}$-integrable (and so $H_{\mathcal{B}}$ -integrable) with the same integral values.

P r o of. Using the above theorem, take $l$ and $u$ for a fixed $\varepsilon$ so that (41) and (5) are fulfilled. We show that $\mathcal{B}$-interval functions $M(I)=\int_{I} l d \mu$ and $m(I)=\int_{I} u d \mu$ are major and minor functions of $f$. Fixing a point $x_{0}$ and using lower semicontinuity of $l$ we can find for any $\eta>0$ a neighborhood $U\left(x_{0}\right)$ of $x_{0}$ such that

$$
l(x) \geq l\left(x_{0}\right)-\eta
$$

for any $x \in U\left(x_{0}\right)$. As $\bigcup_{n=1}^{\infty} C_{n}$ is a base for the topology of $X$, by property (b) of the covers $C_{n}$, we can find $n_{0}$ such that $K\left(n, x_{0}\right) \subset U\left(x_{0}\right)$ for all $n>n_{0}$. So (6) holds for all $x \in K\left(n, x_{0}\right), n>n_{0}$. This implies

$$
\begin{aligned}
\frac{M\left(K\left(n, x_{0}\right)\right)}{\mu\left(K\left(n, x_{0}\right)\right)} & =\frac{1}{\mu\left(K\left(n, x_{0}\right)\right)} \int_{K\left(n, x_{0}\right)} l d \mu \\
& \geq \frac{1}{\mu\left(K\left(n, x_{0}\right)\right)} \int_{K\left(n, x_{0}\right)}\left(l\left(x_{0}\right)-\eta\right) d \mu \\
& =l\left(x_{0}\right)-\eta
\end{aligned}
$$

for the same $n$. From this it follows

$$
\underline{D}_{\mathcal{B}} M\left(x_{0}\right) \geq l\left(x_{0}\right)-\eta \text {. }
$$

By the arbitrariness of $\eta$ and (4) we finally get $\underline{D}_{\mathcal{B}} M\left(x_{0}\right) \geq l\left(x_{0}\right) \geq f\left(x_{0}\right)$. So $M$ is a major function of $f$ for which, by (15), we have

$$
M(X)-\int_{X} f d \mu<\varepsilon .
$$

In a similar way, using upper semi-continuity of $u$, we can show that the $\mathcal{B}$-interval function $m(I)=\int_{I} u d \mu$ is a minor function of $f$ such that

$$
\int_{X} f d \mu-m(X)<\varepsilon .
$$




\section{FRANCESCO TULONE}

Since $\varepsilon$ is arbitrary, (7) and (8) imply that $f$ is $P_{\mathcal{B}}$-integrable on $X$ and the Lebesgue integral $\int_{X} f$ is its $P_{\mathcal{B}}$-integral value on $X$.

Remark 1. Under additional assumption that the measure $\mu$ is non-atomic, the previous construction gives in fact $\mathcal{B}$-continuous major and minor functions. Indeed, under this hypothesis on $\mu$, the absolute continuity of $M$ and $m$ implies their $\mathcal{B}$-continuity. So we have proved by a direct construction that $f$ is also $P_{\mathcal{B}}^{0}$-integrable. Indirectly it follows also from Theorem 4.

\section{REFERENCES}

[1] BOGACHEV, V. I.: Foundations of Measure Theory, Vol. 2. Regular and Chaotic Dynamics, Moscow-Izhevsk, 2003. (In Russian)

[2] GONG, Z. T.: On a problem of Skvortsov involving Perron integral, Real Anal. Exchange $17(1991 / 92)$, 748-750.

[3] LEE, P. Y.-VÝBORNÝ, R.: The Integral: An Easy Approach after Kurzweil and Henstock, in: Austral. Math. Soc. Lect. Ser., Vol. 14, Cambridge University Press, Cambrige, 2000.

[4] LUKASHEnKO, T. P.-SKVORTSOV, V. A.-SOLODOV, A. P.: Generalized Integrals. URSS, Moscow, 2009.

[5] OSTASZEWSKI, K. M.: Henstock integration in the plane, Mem. Amer. Math. Soc. 253 (1986), 1-106.

[6] SAKS, S.: Theory of the Integral. Dover, New York, 1964.

[7] SKVORTSOV, V. A.-TULONE, F.: On the Perron-type integral on a compact zero-dimensional abelian group, Vestnik Moskov. Gos. Univ. Ser. I Mat. Mekh. 72 (2008), 37-42; Engl. transl. in Moscow Univ. Math. Bull. 63 (2008), 119-124.

[8] SKVORTSOV, V. A.-TULONE, F.: Representation of quasi-measure by Henstock-Kurzweil type integral on a compact zero-dimensional metric space, Georgian Math. J. 16 (2009), 575-582.

[9] THOMSON, B. S.: Derivation bases on the real line, Real Anal. Exchange 8 (1982/83), 67-207 and 278-442.

Department of Mathematics and Informatics

University of Palermo

via Archirafi 34

I-90123 Palermo

ITALY

E-mail: tulone@math.unipa.it 